\title{
ATENUAÇÃO DA RADIAÇÃO GAMA NO ESTUDO DA DISTRIBUIÇÃO DE PARTÍCULAS EM SOLO SOB FLORESTA E CANA-DE-AÇÚCAR ${ }^{1}$
}

\author{
J.C.M. de OLIVEIRA ${ }^{2}$; K. REICHARDT ${ }^{3}$ \\ Centro de Energia Nuclear na Agricultura/USP, C.P. 96, CEP:13400-970 - Piracicaba,SP \\ C.M.P. VAZ \\ CNPDIA/EMBRAPA, C.P.741, CEP:13560-970 - São Carlos,SP
}

\begin{abstract}
RESUMO: E apresentado o uso de uma nova metodologia de analise da distribuição de tamanho de partículas de solo, em amostras de terra sob floresta nativa e submetido ao cultivo de cana-de-açúcar por 16, 30 e 50 anos consecutivos. A técnica para análise granulometrica do solo baseia-se na atenuação de um feixe de radiação gama, por uma suspensāo convencional terra/agua em sedimentação. Os resultados indicam uma diferença significativa dos teores de argila nas camadas superficiais do solo em função do cultivo com cana-de-açúcar.

Descritores: análise granulométrica do solo, atenuação da radiação gama, sedimentação, cultivo do solo
\end{abstract}

\section{GAMMA RADIATION ATTENUATION TO STUDY SOIL PARTICLE DISTRIBUTION FOR FOREST AND SUGAR CANE SOILS}

\begin{abstract}
The use of a new soil particle size distribution analysis methodology is presented in connection with the study of particle size distribution in soils covered by natural forest and sugar cane crop cultivated during 16, 30 and 50 years, continuously. The technique is based on the attenuation of a gamma-ray beam by a conventional soil/water suspension under sedimentation. Results show a significant difference in clay content for the upper soil layer as a function of sugar cane cultivation.
\end{abstract}

Key words: soil particle analysis, gamma attenuation, sedimentation, soil management

\section{INTRODUÇÃo}

As partículas do solo após sua análise granulométrica são classificadas, quanto ao tamanho, em três clasşes: argila, silte e areia. Essas partículas podem, por meio de agentes cimentantes, se agrupar formando agregados $\mathbf{e}$ a disposição de partículas e agregados define a estrutura do solo.

A utilização de equipamentos pesados para o manejo das culturas agrícolas, por longos períodos, pode causar a modificação da estrutura do solo, principalmente na sua superfície, afetando a ação de todos os tipos de perdas por migrações de partículas.

O objetivo deste trabalho foi verificar possíveis variações da fração argila de um latosso- lo vermelho escuro, em quatro situações distintas do uso da terra. Uma situação envolve área coberta por vegetação nativa e as outras três, desmatadas há dezesseis, trinta e cinquenta anos, cultivadas exclusiva e continuamente com cana-de-açúcar. Estas situações foram escolhidas, por necessitarem de medidas precisas de tamanho de partículas, sendo ideais para o uso da nova metodologia que emprega a atenuação de um feixe de radiação gama.

\section{MATERIAL E MÉTODOS}

O local do presente estudo situa-se na Usina Capuava S/A, no município de Piracicaba,SP $\left(22^{\circ} 43^{\prime} \mathrm{S} ; 4^{\circ} 38^{\prime} \mathrm{W}\right)$, no sudeste do Brasil. O solo, descrito por CERRI et al. (1991), é um latossolo vermelho escuro, no qual foram indivi-

\footnotetext{
${ }^{1}$ Parte da Teses de Doutorado do primeiro autor, junto ao CENA/USP.

2 Bolsista do FAPESP.

${ }^{3}$ Bolsista do CNPq.
} 
dualizadas quatro situações (tratamentos): uma coberta com vegetação nativa (floresta) e outras três, desmatadas há 16,30 e 50 anos, cultivadas exclusiva e continuamente com cana-de-açúcar. Nas condições da usina, a cada ciclo de quatro anos, toda a cultura é reformada, com arações feitas, inicialmente, por tração animal e, atualmente, por tração mecânica, incluindo sub-solagens. As quatro áreas em estudo distam entre sí, no máximo, $500 \mathrm{~m}$, representando um mesmo solo. As amostragens do solo foram realizadas nas quatro trincheiras, uma em cada tratamento, até $1 \mathrm{~m}$ de profundidade, a cada $10 \mathrm{~cm}$, procurando obter-se amostras compostas representativas. $O$ baixo número de trincheiras limita a análise dos dados face à variabilidade espacial natural dos solos, permite porém a aplicação da nova metodologia, que é o principal objetivo deste trabalho.

Para a obtenção da distribuição de tamanho de partículas do solo foi utilizado um método baseado na atenuação de um feixe de raios gama (VAZ et al., 1992 e OLIVEIRA, 1994). O equipamento utilizado consiste de uma fonte de ${ }^{241} \mathrm{Am}(300 \mathrm{mCi})$, um detector de cristal cintilador de $\mathrm{NaI}(\mathrm{Tl})$ e um conjunto eletrônico padrão para medida e contagem da radiação gama.

No início da sedimentação $(t=t 0=0)$, o feixe de radiação $\epsilon$ posicionado próximo à base do recipiente(ho) e a primeira leitura é feita em um intervalo de tempo $(\Delta t=15 \mathrm{~s})$, pré-estabelecido. $\mathrm{Em}$ seguida, o recipiente é baixado com incrementos de $\Delta \mathrm{h}$, procedendo-se outras leituras $\mathrm{h}$, até à proximidade da superfície da suspensão $\left(h_{n}\right)$. Neste trabalho, cada contagem foi feita em 15s para cada altura $h_{2}(\Delta h=5 \mathrm{~mm})$, em intervalos de 30s. Desta forma, 15 s foram gastos para a contagem do feixe e $15 \mathrm{~s}$ para baixar a amostra de $\Delta \mathrm{h}$. Nestas condições, para um dado tempo $\mathrm{tn}_{\mathrm{n}}>\mathrm{ti}>\mathrm{to}(\mathrm{s})$, após o início da sedimentação, deverão existir apenas partículas de diâmetro menor que $\mathrm{d}_{1}(\mathrm{~cm})$ numa certa altura hi( $\mathrm{cm})$, medida a partir da superfície da solução (equação de Stokes):

$$
\frac{t_{i}}{h_{i}}=\frac{18 \eta}{g d_{i}^{2}\left(D_{p}-D_{p}\right)}
$$

onde $\eta$ (poise) é a viscosidade do líquido; $\mathrm{g}\left(\mathrm{cm} / \mathrm{s}^{2}\right)$ a aceleração da gravidade; $D_{p}$ e $D_{1}\left(g / \mathrm{cm}^{3}\right)$ as densidades das partículas $e$ do líquido, respectivamente.

A concentração $\mathrm{C}(\mathrm{g} / \mathrm{l})$, medida pela atenuação dos raios gama, num dado tempo t,(s) e altura $h_{i}(\mathrm{~cm})$ é dada por:

$$
C=\frac{\ln \left(I_{0} / I\right) 10^{3}}{X\left(\mu_{p}-\mu_{w} D_{w} / D_{p}\right)}
$$

onde $\mathrm{X}(\mathrm{cm})$ é a espessura interna do recipiente atravessada pela radiação; $\mu_{\mathrm{p}}$ e $\mu_{\mathrm{w}}\left(\mathrm{cm}^{2} / \mathrm{g}\right)$ os coeficientes de atenuação de massa do solo e da água, respectivamente e $D_{p}\left(g / \mathrm{cm}^{3}\right)$ e $D_{w}\left(g / \mathrm{cm}^{3}\right)$ a densidade das partículas do solo e da água. As contagens $\mathrm{I}_{0}$ e I (cps) são determinadas pela atenuação do feixe com água e água mais partículas dispersas, dentro do recipiente, respectivamente. Detalhes sobre os procedimentos na medida dos coeficientes de atenuacão e das intensidades de feixe podem ser vistos em OLIVEIRA (1994). Com aquisição dos dados a cada par $h_{1}, t_{1}$, desde o início da sedimentação $(t=t 0=0)$ na base do recipiente $(\mathrm{h} 0)$ até a superfície da solução $\left(h_{n}\right)$, é possível, utilizando-se as equações (1) e (2), determinar a distribuição de tamanho de partículas (OLIVEIRA, 1994).

\section{RESULTADOS E DISCUSSÃo}

O uso da técnica de atenuação de radiação gama permite a obtenção de um maior número de leituras de distribuição de tamanho de partículas, como, por exemplo, pode ser visto nas Figuras 1 e 2 de amostras dos quatro tratamentos, referentes à superfície e à $80 \mathrm{~cm}$ de profundidade.

A diferença marcante da forma da curva referente ao solo superficial de floresta em relação às curvas do solo cultivado (Figura 1), evidencia as diferenças de manejo do solo na variação percentual dos diferentes tamanhos de partículas. Já para as amostras da profundidade de $80 \mathrm{~cm}$, na qual os efeitos do manejo do solo praticamente não se manifestaram, as formas das distribuições são bastante semelhantes (Figura 2).

A título de exemplo na Figura 3 são apresentadas as porcentagens de argila, para as quatro áreas estudadas. Observa-se que a região superficial da área de floresta possui quantidades de argila bem maiores $(50-60 \%)$ que as outras parcelas $(30-40 \%)$.

\section{Calculando-se a razão;}

$$
\frac{I_{z 1}^{22}}{\Delta z}
$$

sendo: 


$$
I_{z 1}^{22}=\int_{z l}^{22} P(z) d z
$$

e $P(z)$ as porcentagens de argila, silte e areia, em função da profundidade, pode-se obter valores médios das frações nos perfis. Os cálculos foram feitos para o perfil total $(\mathrm{zl}=0$ a $\mathrm{z} 2=100 \mathrm{~cm})$ e para duas camadas $(\mathrm{z} 1=0$ a $\mathrm{z} 2=60 \mathrm{~cm}$ e $\mathrm{z} 1=60 \mathrm{~cm}$ a $\mathrm{z} 2=100 \mathrm{~cm}$ ). $O$ limite de $60 \mathrm{~cm}$ de profundidade foi escolhido, pois, aparentemente, as grandes variações nos teores das frações dão-se da superfície até $60 \mathrm{~cm}$ de profundidade. Esses resultados são mostrados na TABELA 1.

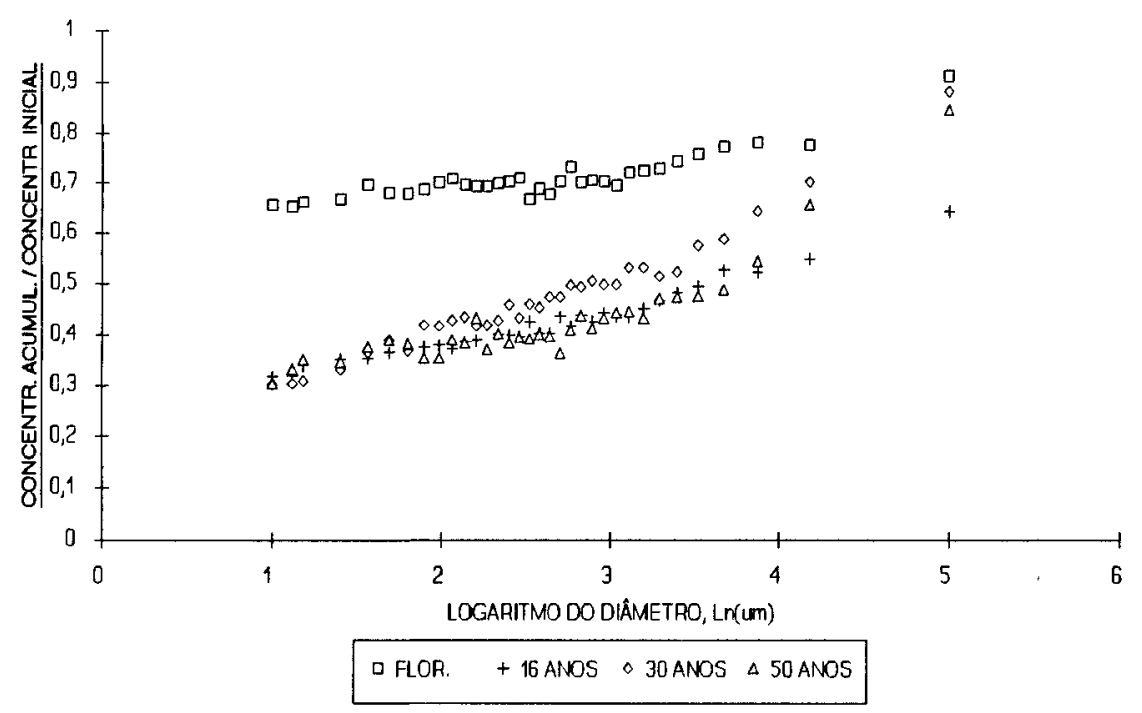

Figura 1 - Curvas da distribuição de tamanho de partículas do solo, da camada superficial, para as quatro áreas estudadas.

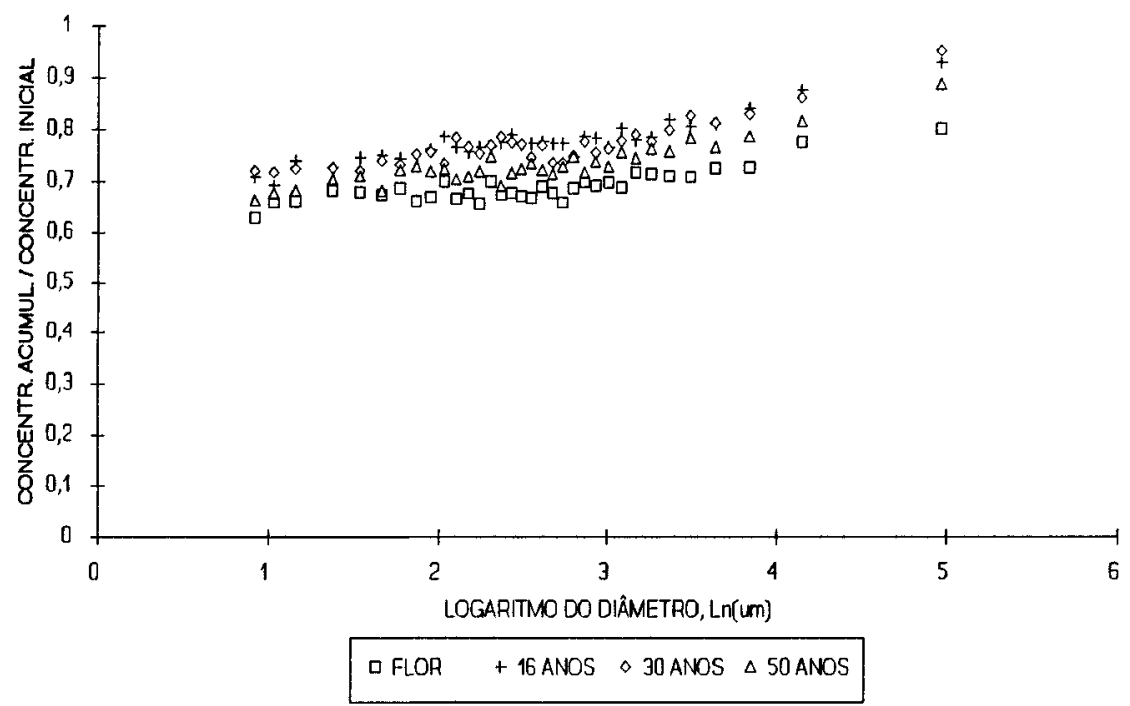

Figura 2 - Curvas da distribuição de tamanho de partículas do solo, da camada de $80 \mathrm{~cm}$ de profundidade, para as quatro áreas estudadas. 


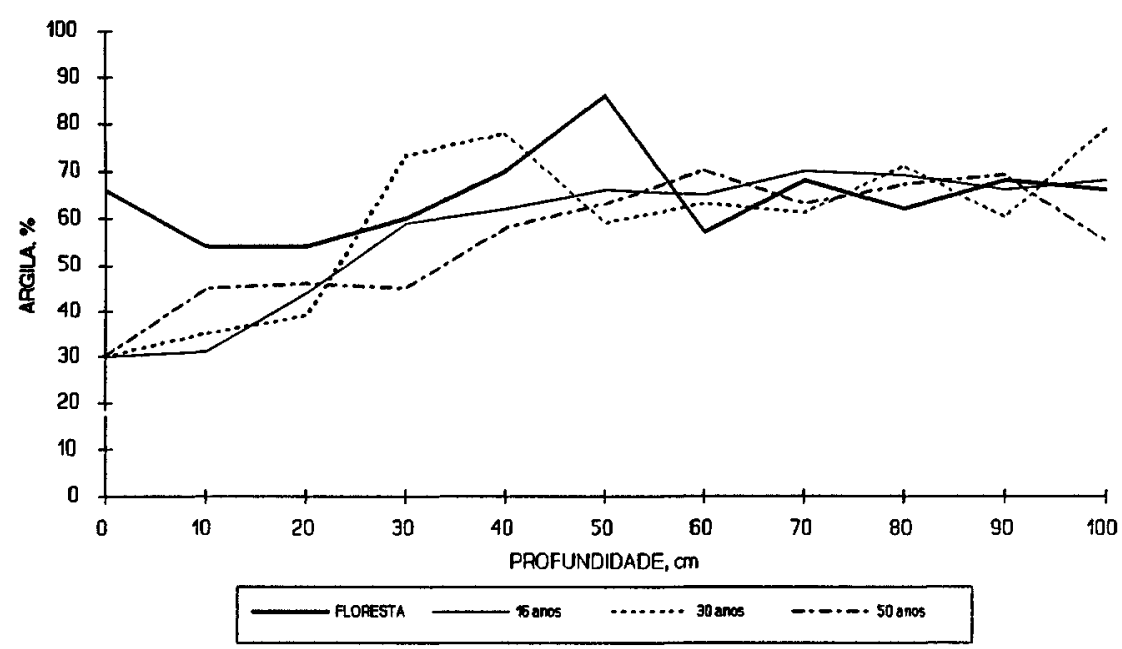

Figura 3 - Porcentagens de argila ao longo do perfil, para as quatro áreas estudadas.

TABELA 1. Valores médios das porcentagens de argila, silte $e$ areia total, nos intervalos de 0 a 100,0 a 60 e 60 a $100 \mathrm{~cm}$ de profundidade.

\begin{tabular}{cccc}
\hline Área & $\mathrm{I} / \Delta \mathrm{z}$ & $\mathrm{I} / \Delta \mathrm{z}$ & $\mathrm{I} / \Delta \mathrm{z}$ \\
& $0-100 \mathrm{~cm}$ & $0-60 \mathrm{~cm}$ & $60-100 \mathrm{~cm}$
\end{tabular}

Argila Silte Areia Argila Silte Areia Argila Silte Areia $\begin{array}{llllllllll}\text { Floresta } & 65 & 13 & 21 & 64 & 14 & 20 & 65 & 11 & 21\end{array}$ $\begin{array}{llllllllll}16 \text { anos } & 58 & 16 & 27 & 57 & 17 & 31 & 68 & 13 & 20\end{array}$

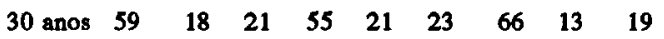
$\begin{array}{llllllllll}50 \text { anos } & 57 & 16 & 27 & 51 & 18 & 31 & 65 & 13 & 22\end{array}$

Observou-se, desses resultados, que a quantidade acumulada de argila em todo o perfil diminui de $65 \%$ (floresta) para $58 \%, 59 \%$ e $57 \%$, respectivamente, para as áreas de cultivo de 16,30 e 50 anos. No entanto, nota-se que de 60 a $100 \mathrm{~cm}$ as quantidades acumuladas de argila mantiveram-se praticamente constantes, com pequenos aumentos de $65 \%$ (floresta) para $68 \%, 66 \%$ e $65 \%$ para as áreas de 16,30 e 50 anos, respectivamente. Já entre a superfície e $60 \mathrm{~cm}$, as quantidades acumuladas de argila diminuíram mais acentuadamente: de $64 \%$ (floresta) para $57 \%, 55 \%$ e $51 \%$, respectivamente para 16, 30 e 50 anos de cultivo.

Com relação às quantidades de limo e areia, aparentemente, não houve mudanças signifi- cativas. Os pequenos aumentos e decréscimos podem ser atribuídos às variações percentuais das quantidades de argila e a erros de amostragem.

De acordo com os resultados obtidos, pode-se concluir que houve variações dos teores de argila nas camadas superficiais, causadas pelo manejo do solo. Há evidência de uma relação entre tempo de cultivo de cana-de-açúcar nestas áreas e as variações observadas.

\section{REFERÊNCIAS BIBLIOGRÁFICAS}

CERRI, C.C.; FELLER, C.; CHAUVEL, A. Evolução das principais propriedades de um latossolc vermelho escuro após desmatamento e cultivo por doze e cinquenta anos com cana-de-açúcar. Cahiers Orstom. Série Pédologie, Paris, v.26, n.1, p.37-50, 1991.

OLIVERRA, J.C.M. de. Atenuação de raios gama na análise granulométrica de amostras de terra. Piracicaba, 1994. 67p. Tese (Doutorado). Centro de Energia Nuclear na Agricultura, Universidade de São Paulo.

VAZ, C.M.P.; OLIVEIRA, J.C.M.; REICHARDT, K.; CRUVINEL, P.E.; BACCHI, O.O.S. Soil mechanical analysis through gamma ray attenuation. Soil Technology, Cremligen, v.5, n.4, p.319-325, 1992.

Recebido para publicação em 02.03.94

Aceito para publicação em 20.03.95 